\title{
CONGENITAL KYPHOSIS IN MYELOMENINGOCELE
}

\author{
Stanley Hoppenfeld, New York, United States of America \\ From the Children's Hospital and the University Department of Orthopaedics, Sheffield, England
}

More children with spina bifida cystica are surviving today because of improved operative treatment and clinical care. Problems arising from spinal deformity in these children are presenting more often for treatment.

The purpose of this paper is to observe the natural course of kyphotic spinal deformity seen in association with spina bifida cystica. Specific attention is focused on kyphosis because of the unique difficulties that present both at birth and in later childhood, and because of its possible neurological complications.

\section{MATERIAL}

Radiographs were studied of all patients with meningomyelocele who were admitted to the Sheffield Children's Hospital between 1956 and 1960 for early closure of the meningomyelocele and who survived. There were 211 survivors during this period, and radiographs of 197 of them were available for review. The range of follow-up was between five and nine years. Two hundred and eighty-three patients out of 500 currently attending a spina bifida and meningomyelocele follow-up clinic were also examined. In addition, observations were made on thirty-one newborn patients at operation to close a spinal defect associated with meningomyelocele. Of these, five had a congenital kyphotic deformity. Dissections were made of necropsy material from four newborn infants not yet fixed in formalin and of seven newborn infants fixed in formalin. All these infants had died within the first two weeks of life. Dissection was carried out on one patient who had died at three years of age.

\section{CLINICAL OBSERVATIONS}

Spina bifida with meningomyelocele is associated with kyphosis at birth in one in every eight cases. The kyphosis is fixed and is not altered when the child is prone or supine. This contrasts with infants with spina bifida and no true kyphosis, in whom the area of spine involved is supple. The kyphosis is usually in the lumbar spine and is usually restricted to the area of the spinal defect. The pedicles are present and protrude postero-laterally, accentuating the kyphotic appearance.

Children with meningomyelocele sit up at a later age than do normal children. When they do sit up, the fixed kyphosis is retained and often increased by the weight of the body. In the sitting position the patient develops a compensatory thoracic lordosis just above the fixed kyphosis, and the angle of this is sometimes quite acute. In contrast, children with a paralysed lumbar spine, but without congenital kyphosis, show lumbar lordosis when they sit, which may become severe, fixed and associated with scoliosis.

Those children who had fixed kyphosis at birth and eventually walked, aided or unaided, did not lose the kyphosis when walking. The deformity tends to increase with growth and weight bearing, and any increase may be associated with increasing neurological involvement shown by further paralysis of the lower limbs and bladder. The deformity adds to the difficulties of bracing if calipers with hip hinges and trunk support are needed.

\section{OBSERVATIONS AT OPERATION}

At the time of operation for primary closure the neural plaque is freed from the surrounding membrane and the membrane is excised. The open dura is then visible. It lies 
over the posterior aspect of the vertebral bodies and continues laterally over the pedicles. The dura is dissected free from the pedicles for closure around the cord. The pedicles are then exposed. They project postero-laterally and present an additional surgical problem in closure of the skin. Though the skin can usually be closed by extensive mobilisation to give a mid-line suture, the pedicles act as pressure points over which the skin is blanched, and skin necrosis may develop. If the suture line heals, the pedicles continue to act as pressure points so that the skin may break down at any time. This will occur if continual pressure is applied over these prominences when the child is lying, sitting or wearing a brace (Fig. 1).

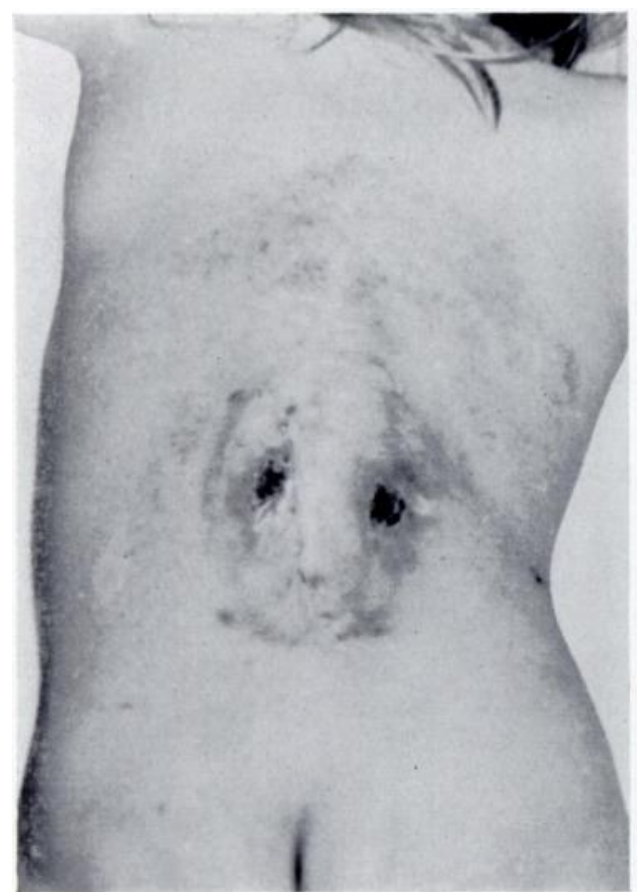

Fig. 1

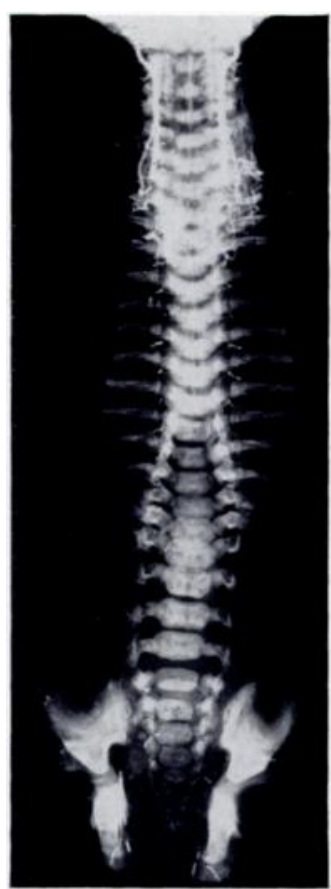

Fig. 2

Figure 1-A child aged 2 years showing kyphosis of the spine with two areas of skin necrosis on either side of the mid-line caused by pressure on skin overlying the prominent bony pedicles. Figure 2-Radiograph of a dissected axial skeleton of a newborn child with a thoraco-lumbo-sacral defect. It shows the fusiform pattern made by the widespread pedicles and the narrowed intervertebral joint spaces in the area of kyphosis.

Direct pressure applied to the apex of the kyphosis at the time of primary operation reveals that the kyphosis may be springy but not reducible. In one case the deformity was partly reduced after pressure was applied, and a snap was heard as if an adhesion had been broken. However, no means could be devised to maintain the reduction, and altered skin sensation did not allow external fixation and correction by plaster. Because of the extent of the kyphosis the condition of the skin was precarious and it subsequently broke down. The patient died five days later from meningitis. At necropsy it was found that the anterior longitudinal ligament had been stretched at the apex of the kyphosis to allow some mobility. There was no damage to the overlying vascular structures.

\section{RADIOLOGICAL OBSERVATIONS}

Antero-posterior and lateral radiographs of the axial skeleton of the newborn child with spina bifida and meningomyelocele reveal the defects that are present in congenital kyphosis. 
The antero-posterior view demonstrates that the spinous processes are absent, and the laminae are absent or rudimentary as characteristic of spina bifida. The pedicles of the individual vertebra are splayed and present a fusiform pattern extending from the eleventh thoracic vertebra to the sacrum. The pedicles are widest apart at the apex of the kyphos. The intervertebral spaces are narrower than normal, the change being most marked at the apex of the curve. The vertebral bodies appear to overlap (Fig. 2). Though usually well formed, some vertebral bodies showed congenital divisions with two centres of ossification. The bodies appear to be wider than normal but radiographs of individual vertebrae did not substantiate this (Figs. 3 and 4).

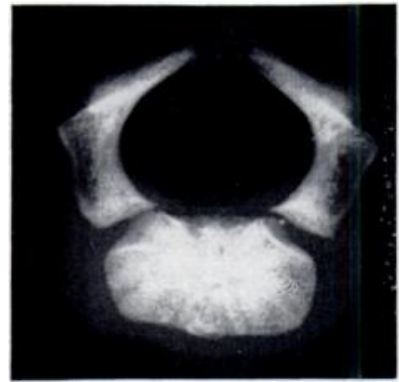

FIG. 3

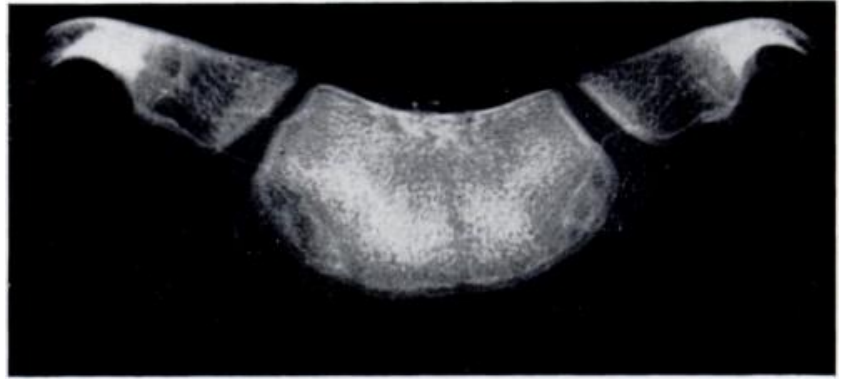

FIG. 4

Figure 3-A normal vertebra of a newborn child. Figure 4-Vertebra associated with severe spina bifida and fixed kyphosis. The neurocentral epiphysial line has changed its angle and the interpedicular space is widened.

When kyphosis is present the lower ribs are seen to be elevated from their normal downward angulation to a more horizontal position, producing a crowning effect (Fig. 5). Many children had antero-posterior films available from excretory urography. Even without lateral films some information can be obtained. Diagnosis of kyphosis of the lumbar spine may be made from the antero-posterior view when the spina bifida extends from the eleventh thoracic level downwards to include the sacrum, by observing the widening of the interpedicular space, elevation of the lower ribs and narrowing of the intervertebral spaces.

In the lateral film the kyphosis of the lumbar area is easily seen. The vertebral bodies are narrowed anteriorly, most at the apex (Figs. 6 and 7). The intervertebral spaces are narrowed. The spaces are narrowed more anteriorly than posteriorly and may be completely obliterated at the apex of the curve. The spinous process and laminae are not seen. The facets of the zygoapophysial joints in the area of fixed lumbar kyphosis are seen in the coronal plane instead of the normal sagittal plane.

\section{NECROPSY OBSERVATIONS}

Bone-The spinal columns from twelve cases of spina bifida with meningomyelocele were studied. Eleven patients had died within the first two weeks of life, and the twelfth died at the age of three. Of these, ten had a fixed kyphotic deformity and two had no fixed kyphosis.

When fixed kyphosis was present, the pedicles were widely spread in the area of deformity and less so in the adjacent areas. The posterior ends of the pedicles were more laterally placed than the anterior ends attached to the vertebrae. The pedicles were attached to the vertebral body through a cartilaginous neurocentral junction (Fig. 4). The ascending and descending facets were present and articulated with each other. At occasional levels the articular surfaces fused with each other forming a continuous lateral cartilaginous linkage. The articular processes and their pedicles, in conjunction with the posterior aspects of the adjacent vertebral bodies, formed a foramen for the nerve roots. The facets in the kyphotic lumbar area articulated with each other in the coronal plane of the body instead of the normal sagittal plane. This is 
explained by the fact that although the articular process and facets maintain their normal relationship to the pedicles, the pedicles have moved or rotated laterally, so altering the plane of the articulation of the facets from sagittal to coronal (Fig. 8).

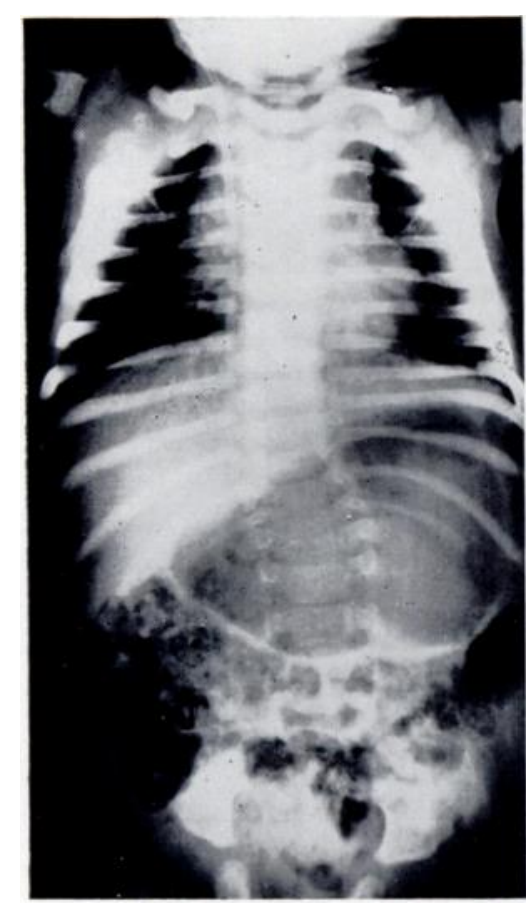

FiG. 5

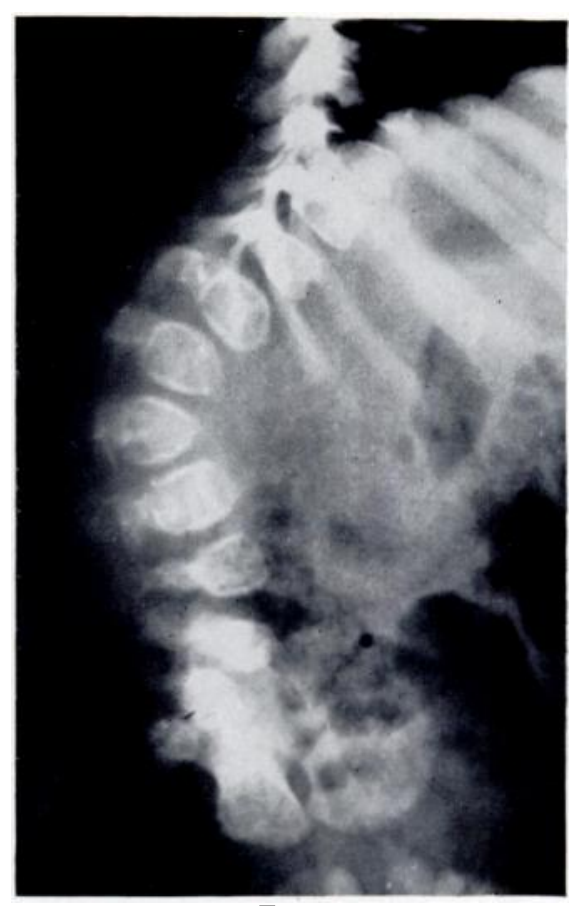

FIG. 6

Figure 5-Radiograph of a newborn child with kyphosis, to show the elevation of the lower ribs producing a crowning effect and the narrowed intervertebral joint spaces. Figure 6-Lateral radiograph in the sam ecase illustrating the kyphosis and wedging of the apical vertebrae within the deformity.

Soft tissues-The annulus fibrosus was contracted or tightened anteriorly in association with the narrowed anterior intervertebral space. The nucleus pulposus was located posteriorly at the apex of the deformity. The anterior longitudinal ligament was attached to the anterior surface of the bodies and followed the kyphotic curvature of the spine. It was shortened as it crossed the narrowed anterior intervertebral space. In two specimens there was a marked cord-like thickening or cicatrisation in the anterior longitudinal ligament, attached to the vertebral bodies and spanning the deformed section of the spine (Fig. 9). Sectioning the cicatrices partly corrected the kyphosis. Section of the annulus fibrosus allowed still greater correction of the kyphotic deformity on the specimens (Fig. 10). No correction was achieved by section of the anterior longi-

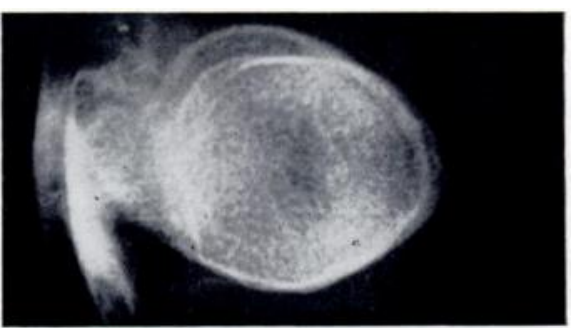

FIG. 7

Radiograph of a vertebra at the apex of the kyphosis, showing that its centre of ossification is compressed and rounded anteriorly. tudinal ligament in the remaining specimens but sectioning of the annulus fibrosus resulted in mobilisation of the deformity. The aorta followed the anterior surface of the vertebral bodies. It was not kinked and was not adherent to the vertebral bodies or anterior longitudinal ligament.

The spinal membranes had been closed surgically in all cases and the closure corresponded to the area of spinal deformity. The cord was found to be mobile within the deformed area, 


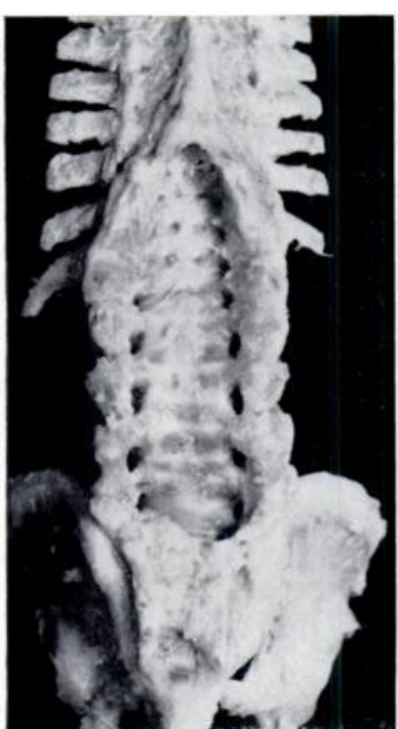

FIG. 8

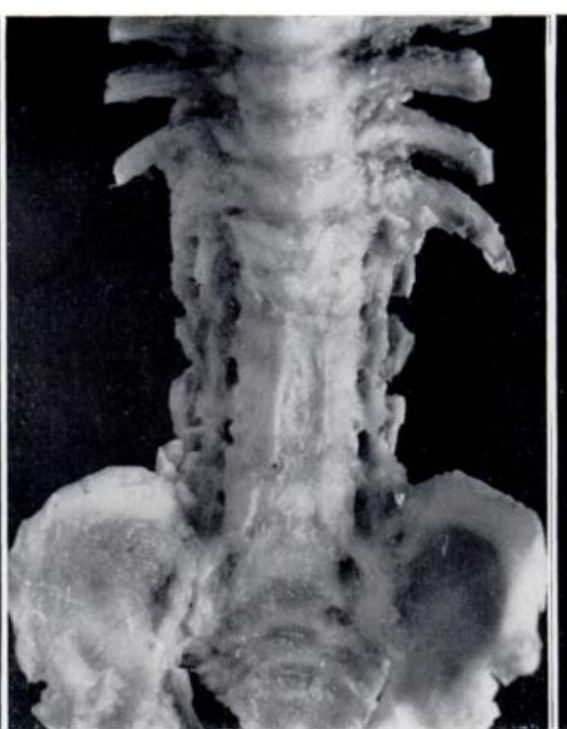

FIG. 9

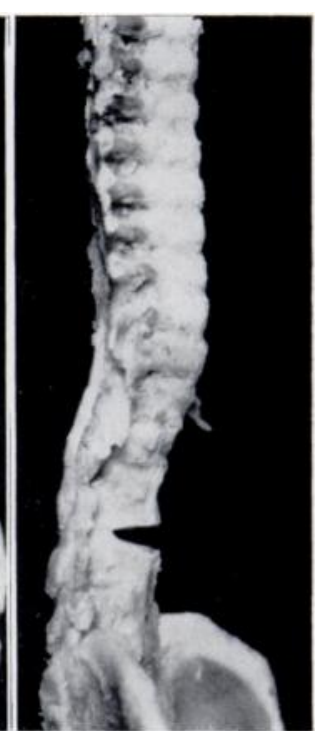

FIG. 10

Figure 8-Posterior vicw of a dissected specimen. The facets in the lumbar area are seen to articulate in the coronal plane instead of the sagittal plane. Some of the facets have fused with each other forming a continuous lateral cartilaginous link. Figure 9-Anterior view of dissected specimen showing the deformity in the lumbar region and the cicatrix formation in the anterior longitudinal ligament. Figure 10-Lateral view of a dissected specimen showing how correction of the kyphosis has been obtained by cutting the cicatrix, anterior longitudinal ligament and anterior portion of the annulus fibrosus at the apex of the deformity.

but in one specimen, from the patient aged three, diastematomyelia limited the mobility of the cord (Fig. 11).

The nerve roots left the canal close to the posterior surface of the bodies, and were not obstructed by bony deformity. The paraspinal muscles were laterally displaced and were found

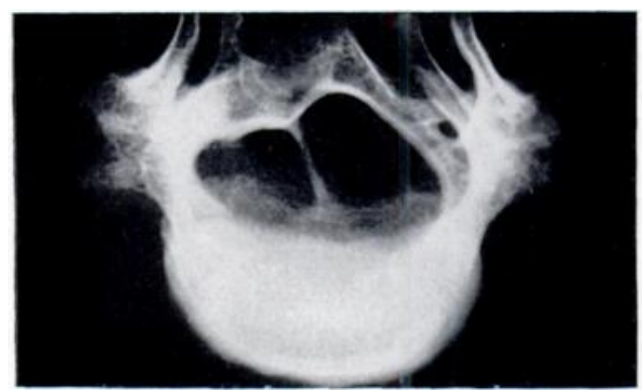

FIG. 11

Axial radiographs of lumbar vertebrae showing diastematomyelia in a patient aged 3 years with meningomyelocele and spina bifida. next to the pedicles. The transverse process was present and separated the psoas muscles from the displaced paraspinal muscles. The residual or rudimentary laminae were found to be of cartilage and completely angulated laterally, so that their original anterior surface was now facing posteriorly (Fig. 4).

The two patients with supple spines and no kyphotic deformity revealed no contractures of the anterior longitudinal ligament. The nucleus pulposus was centrally located. The pedicles were only slightly spread and the lumbar facets articulated in the sagittal plane of the body.

\section{TREATMENT}

It is not the purpose of this paper to discuss the treatment of this deformity, but it is obvious that any safe means of obtaining even partial correction of the deformity at the initial operation on the spine would considerably ease the problems of skin closure and diminish the secondary complications that follow. This, and the means to prevent progressive increase in deformity with growth and weight bearing, warrant further study.

This study was performed while I was at the Sheffield Children's Hospital under the tutelage of Mr W. J. W. Sharrard. The scholarship was provided by the Frauenthal Fellowship, Hospital for Joint Diseases, New York City, New York. I wish to express my appreciation of the staff of the surgical, pathological, radiological and photographic departments at the Children's Hospital for their kind assistance in this work. 